\title{
MULTIPLIERS OF HILBERT SPACES OF ANALYTIC FUNCTIONS ON THE COMPLEX HALF-PLANE
}

\author{
ANDRZEJ STANISŁAW KUCIK
}

\begin{abstract}
It follows, from a generalised version of Paley-Wiener theorem, that the Laplace transform is an isometry between certain spaces of weighted $L^{2}$ functions defined on $(0, \infty)$ and (Hilbert) spaces of analytic functions on the right complex half-plane (for example Hardy, Bergman or Dirichlet spaces). We can use this fact to investigate properties of multipliers and multiplication operators on the latter type of spaces. In this paper we present a full characterisation of multipliers in terms of a generalised concept of a Carleson measure. Under certain conditions, these spaces of analytic functions are not only Hilbert spaces but also Banach algebras, and are therefore contained within their spaces of multipliers. We provide some necessary as well as sufficient conditions for this to happen and look at its consequences.
\end{abstract}

Mathematics subject classification (2010): Primary 30H50, 46J15, 47B99; Secondary 46E22, 46J20.

Keywords and phrases: Banach algebras, Banach spaces, Bergman spaces, Carleson measures, Dirichlet spaces, Hardy spaces, Hardy-Sobolev spaces, Hilbert spaces, Laplace transform, maximal ideal spaces, multiplication operators, multipliers, reproducing kernels, spaces of analytic functions, weighted $L^{2}$ spaces, Zen spaces.

\section{REFERENCES}

[1] H. A. Alsaker, Multipliers of the Dirichlet space, Master's Thesis in Mathematical Analysis, Department of Mathematics, University of Bergen, Norway, 2009.

[2] N. ARONSZAJn, Theory of reproducing kernels, Transactions of the American Mathematical Society 683 (1950), 337-404.

[3] F. F. Bonsall and J. Duncan, Complete Normed Algebras, Springer-Verlag, 1973.

[4] A. Borichev ANd H. Hedenmalm, Completeness of translates in weighted spaces on the half-line, Acta Mathematica 174 (1995), 1-84.

[5] L. CARLESON, Interpolations by Bounded Analytic Functions and the Corona Problem, Annals of Mathematics 763 (1962), 547-559.

[6] I. Chalendar And J. R. Partington, Norm Estimates for Weighted Composition Operators on Spaces of Analytic Functions, Complex Analysis and Operator Theory 85 (2013), 1087-1095.

[7] C. C. Cowen And B. D. MacCluer, Composition Operators on Spaces of Analytic Functions, CRC Press, Studies in Advanced Mathematics, 1995.

[8] H. G. DALes, Banach Algebras and Automatic Continuity, Clarendon Press, Oxford Science Publications, London Mathematical Society Monographs New Series 24, 2000.

[9] P. L. Duren, Theory of $H^{p}$ spaces, Academic Press, 1970.

[10] P. L. Duren And A. Schuster, Bergman Spaces, American Mathematical Society, Mathematical Surveys and Monographs 100, 2004.

[11] P. L. Duren, E. A. Gallardo-Gutiérrez and A. Montes-Rodríguez, A Paley-Wiener theorem for Bergman spaces with application to invariant subspaces, Bulletin of the London Mathematical Society 393 (2007), 459-466.

[12] O. El-Fallah, K. Kellay, J. Mashreghi and T. Ransford, A Primer on the Dirichlet Space, Cambridge University Press, Cambridge Tracts in Mathematics 203, 2014.

[13] T. W. Gamelin, Uniform Algebras, Prentice-Hall, Inc., Prentice-Hall Series in Modern Analysis, 1969. 
[14] D. J. H. Garling, Inequalities: A Journey into Linear Analysis, Cambridge University Press, 2007.

[15] J. B. Garnett, Bounded Analytic Functions, Academic Press, 1981.

[16] E. A. Gallardo-Gutiérrez, J. R. Partington And D. Segura, Cyclic vectors and invariant subspaces for Bergman and Dirichlet shifts, Journal of Operator Theory 621 (2009), 199-214.

[17] Z. HARPER, Boundedness of Convolution Operators and Input-Output Maps Between Weighted Spaces, Complex Analysis and Operator Theory 31 (2009), 113-146.

[18] Z. HARPER, Laplace Transform Representations and Paley-Wiener Theorems for Functions on Vertical Strips, Documenta Mathematica 15, (2010), 235-254.

[19] H. Hedenmalm, B. Korenblum And K. Zhu, Theory of Bergman Spaces, Springer, Graduate Texts in Mathematics 199, 2000.

[20] K. Hoffman, Banach Spaces of Analytic Functions, Prentice-Hall International, Prentice-Hall Series in Modern Analysis, 1962.

[21] B. JaCOB And J. R. PARTington, Admissibility of Control and Observation Operators for Semigroups: A Survey, Operator Theory: Advances and Applications 149, (2004), 199-221.

[22] B. Jacob, J. R. PARtington And S. Pott, On Laplace-Carleson embedding theorems, Journal of Functional Analysis 2643 (2013), 783-814.

[23] B. JaCoB, J. R. PARTington And S. Pott, Applications of Laplace-Carleson Embeddings to Admissibility and Controllability, SIAM Journal of Control and Optimization 522 (2014), 1299-1313.

[24] Yu. N. KuZnetsova, Weighted $L_{p}$-Algebras on Groups, Functional Analysis and Its Applications 403 (2006), 234-236.

[25] J. Mashreghi, Representation Theorems in Hardy Spaces, Cambridge University Press, London Mathematical Society Student Texts 74, 2009.

[26] N. K. NiKOLSKII, Spectral synthesis for a shift operator and zeros in certain classes of analytic functions smooth up to the boundary, Soviet Mathematics (translated from Doklady Akademii Nauk SSSR) 111 (1970), 206-209.

[27] V. I. Paulsen, M. Raghupathi, An Introduction to the Theory of Reproducing Kernel Hilbert Spaces, Cambridge University Press, 2016.

[28] C. E. RickART, General Theory of Banach Algebras, Robert E. Krieger Publishing Company, 1960.

[29] D. A. Stegenga, Multipliers of the Dirichlet space, Illinois Journal of Mathematics 241 (1980), 113-139.

[30] D. V. Widder, The Laplace Transform, Princeton University Press, 1941. 